\title{
Deciphering the flow structure of Czochralski melt using Partially Averaged Navier-Stokes (PANS) method
}

\author{
SUDEEP VERMA ${ }^{1,2}$ and ANUPAM DEWAN ${ }^{2, *}$ \\ ${ }^{1}$ Solid State Physics Laboratory, Defence Research and Development Organisation (DRDO), \\ Timarpur, Delhi 110054, India \\ ${ }^{2}$ Department of Applied Mechanics, Indian Institute of Technology Delhi, New Delhi 110016, India \\ e-mail: adewan@am.iitd.ac.in
}

MS received 25 April 2017; revised 1 August 2017; accepted 30 August 2017; published online 5 February 2018

\begin{abstract}
Czochralski melt flow is an outcome of complex interactions of centrifugal, buoyancy, coriolis and surface tension forces, which act at different length and time scales. As a consequence, the characteristic flow structures that develop in the melt are delineated in terms of recirculating flow cells typical of rotating BénardMarangoni convection. In the present study, Partially Averaged Navier-Stokes (PANS) method is used for the first time to study an idealized Czochralski crystal growth set-up. It is observed that with a reduction in the PANS filter width, more turbulent scales are resolved and the present PANS model is able to resolve almost all the characteristic flow structures in the Czochralski flow at a comparatively lower computational cost compared with more advanced turbulence modelling tools, such as Direct Numerical Simulation (DNS) and Large Eddy Simulation (LES).
\end{abstract}

Keywords. Czochralski flow; Large Eddy Simulation; Direct Numerical Simulation; Partially Averaged Navier-Stokes method.

\section{Introduction}

Czochralski technique is one of the most sought after techniques for the growth of large size semiconductor single crystals, which involves pulling of single crystal from the melt held in a heated crucible using a seed crystal. The crystal and crucible are usually rotated in opposite directions for homogenizing the thermal and solutal fluctuations in the melt. Non-linear interactions of various forces in Czochralski set-up cause different flow instabilities, resulting in flow anisotropy and making it turbulent $[1,2]$. The resolution of this flow characteristics using the conventional two-equation Reynolds-Averaged Navier-Stokes (RANS) models, such as, the standard $k-\varepsilon$ model, is not possible due to the assumptions of an isotropic eddy viscosity and inherent averaging involved [3]. Hence advanced turbulence modelling approaches, such as, Direct Numerical Simulation (DNS) and Large Eddy Simulation (LES), have been used for a comprehensive Czochralski flow analysis. These approaches resolve/model the smallest of the fluctuations in space and time but require extremely fine mesh and therefore quite large computational resources (see table 1). Recently, Verma and Dewan [8] presented a comprehensive review of significant contributions and different modelling approaches used for Czochralski melt flow analysis.

*For correspondence
In recent times, the Partially Averaged Navier-Stokes (PANS) approach proposed by Girimaji [9] has gained significant attention of researchers working in the field of turbulence modelling due to its unique ability to resolve complex flow structures using a reasonably smaller grid size. The PANS method has been quite successful in resolving a variety of turbulent flows, such as flow over a bluff body [10], turbulent thermal plume [11], flow inside a cavity [12], etc. In the present study, the PANS method has been applied for the first time to analyse the fluid flow characteristics in an idealized Czochralski set-up, which represents a rotating flow driven by buoyancy and surface tension.

\section{Problem description and model formulation}

Figure 1a shows the idealized Czochralski set-up used in the present study with the crystal and crucible rotating at $20 \mathrm{rpm} \mathrm{CW}\left(\omega_{c}\right)$ and $5 \mathrm{rpm} \mathrm{CCW}\left(\omega_{s}\right)$, respectively, $R_{i}=50 \mathrm{~mm}, R_{c}=170 \mathrm{~mm}$ and $H=85 \mathrm{~mm}$. The thermophysical properties of $\mathrm{Si}$ as described in [5] were used.

No-slip boundary conditions were applied on the crucible walls and crystal surface. The temperature at the crystal was held constant at $1685 \mathrm{~K}$; the melting point of Si $\left(T_{\text {min }}\right)$ and the temperature profiles at the vertical and bottom walls $T(z)$ and $T(r)$ were fixed according to the experimental conditions of Gräbner et al [13] given by the expressions 
Table 1. Summary of significant contributions on Czochralski flow structure analysis (with crystal and crucible rotations).

\begin{tabular}{lccc}
\hline Reference & Year & Grid & Method \\
\hline Raufeisen et al [4] & 2009 & $10,48,576$ & LES \\
Raufeisen et al [5] & 2008 & $83,88,608$ & DNS \\
Wagner and Friedrich [6] & 2004 & $28,40,064$ & DNS \\
Kumar et al [7] & 2003 & $10,00,000$ & LES \\
Enger et al [14] & 2001 & $19,45,600$ & DNS \\
\hline
\end{tabular}

$$
\begin{aligned}
\frac{T(z)-T_{0}}{\Delta T}= & 4.6\left(\frac{H-z}{R_{c}}\right)^{3}-6.85\left(\frac{H-z}{R_{c}}\right)^{3}+3.615 \frac{H-z}{R_{c}} \\
& -0.619 \text { and }
\end{aligned}
$$

$$
\frac{T(r)-T_{0}}{\Delta T}=0.619\left(\frac{r}{R_{c}}\right)^{2}-0.119,
$$

where $T_{0}=\left(T_{\max }+T_{\min }\right) / 2$ and $\Delta T=T_{\max }-T_{\min }$.

Here $T_{\max }$ denotes the maximum temperature in the computational domain, which occurred at the bottom corner of the crucible. Marangoni convection was taken into account on the free-surface, which was assumed to be a flat surface with radiative heat transfer to the surroundings. Figure $1 \mathrm{~b}$ shows different forces acting on the Czochralski melt flow and in figure 1c the blockstructured grid consisting of 14 blocks with 6,82,560 mesh elements used in the present study is shown. Table 2 presents the values of important dimensionless numbers for the present study. Values of the Reynolds number and the Rayleigh number in the present study (table 2) are of the order of $10^{4}$ and $10^{7}$, respectively, which are quite close to the turbulent flow regime. Therefore, the Czochralski flow is expected to be three-dimensional with large scale fluctuations.

The present PANS model was validated with the experimental and DNS results of Enger et al [14] and Wagner and Friedrich [6].

The partial averaging in the PANS method is similar to the RANS modelling, and the modelling of the sub-filterstress (SFS) is also performed using a Boussinesq-type approximation employing the unresolved PANS eddy viscosity. The PANS approach belongs to a group of variable resolution methods wherein the accuracy or resolution of the solution can be varied from the RANS modelling to DNS depending upon the value of the resolution control parameter/cutoff-filters $f_{k}$ and $f_{\varepsilon}$. They are defined as the ratio of the unresolved turbulent kinetic energy $\left(k_{u}\right)$ to total kinetic energy $(k)$ and the unresolved dissipation $\left(\varepsilon_{u}\right)$ to total dissipation $(\varepsilon)$, respectively:

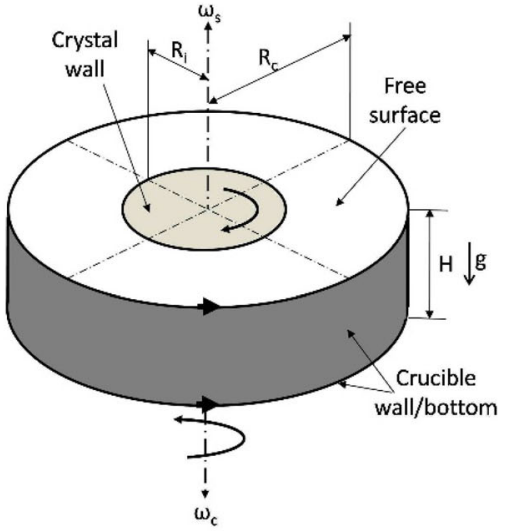

(a)

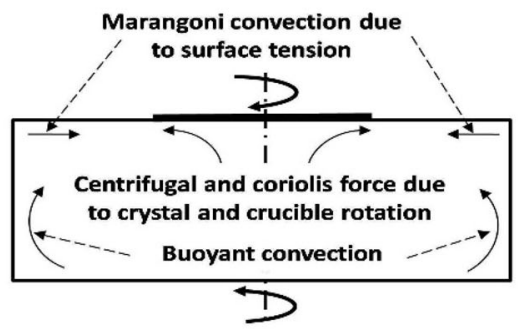

(b)

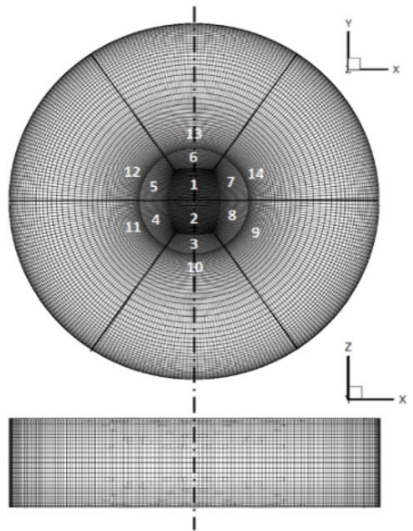

(c)

Figure 1. (a) Ideal Czochralski geometry used in the present study, (b) different forces acting on the Czochralski melt (top thick line represents the crystal boundary) and (c) block-structured grid used in the computational domain.

Table 2. Definitions and values of important dimensionless numbers.

\begin{tabular}{lcc}
\hline Dimensionless number & Symbol & Value \\
\hline Prandtl number & $\operatorname{Pr}$ & $1.284 \times 10^{-2}$ \\
Reynolds number & $\operatorname{Re}$ & $4.697 \times 10^{4}$ \\
Reynolds number (rotational) & $\operatorname{Re}_{w}$ & $4.451 \times 10^{4}$ \\
Grashof number (thermal) & $\mathrm{Gr}$ & $2.206 \times 10^{9}$ \\
Marangoni number & $\mathrm{Ma}$ & $2.822 \times 10^{4}$ \\
Rayleigh number & $\mathrm{Ra}$ & $2.833 \times 10^{7}$ \\
Taylor number & $\mathrm{Ta}$ & $7.923 \times 10^{9}$ \\
\hline
\end{tabular}




$$
f_{k}=\frac{k_{u}}{k} \text { and } f_{\varepsilon}=\frac{\varepsilon_{u}}{\varepsilon} .
$$

The parameters $f_{k}$ and $f_{\varepsilon}$ control the extent of averaging in a PANS model. With $f_{k}=1$, the entire turbulent kinetic energy of the flow field is unresolved (modelled) and the PANS model would render a RANS solution. On the other hand, setting $f_{k}=0$ on a sufficiently fine grid results in the resolution of all the turbulent kinetic energy, thus obtaining a DNS solution. To find out the value of $f_{k}$, Girimaji and Hamid [15] proposed a method based on the Taylor turbulence length scale and grid dimensions. According to [15], the value of $f_{k}$ that a grid can support is given by the expression

$$
f_{k}=\frac{1}{\sqrt{C_{\mu}}}\left(\frac{\Delta}{\Lambda}\right)^{2 / 3}
$$

where $C_{\mu}=0.09, \Delta=\left(\Delta_{x} \Delta_{y} \Delta_{z}\right)^{1 / 3}$ is the average cell dimension and $\Lambda=k^{3 / 2} / \varepsilon$ is the turbulent length scale computed by performing a RANS simulation prior to starting the PANS computations on the same mesh.

The partially averaged equations for mass, momentum and energy along with the low-Re $k-\varepsilon$ equations in terms of $k_{u}$ and $\varepsilon_{u}$ (obtained after multiplying the parent RANS low$R e k-\varepsilon$ equations with $f_{k}$ and $f_{\varepsilon}$, respectively) and the PANS modified model constants used in the present analysis are described in [16]. We have used the low-Re $k-\varepsilon$ model of Launder and Sharma and therefore the damping functions and model constants for the parent RANS low-Re Launder and Sharma $k-\varepsilon$ model have been used [17]. In addition, the Boussinesq approximation and buoyancy correction have been applied to the momentum and $k_{u}$ and $\varepsilon_{u}$ equations, respectively [18]. Additional source terms for incorporating the effect of centrifugal and coriolis forces were included in the momentum equations [4].

The PANS technique is highly sensitive to the type of discretization scheme used and may induce spurious oscillations in the solution, which can be easily confused with more resolved fluctuations. Hence the equations were discretized by the finite-volume method (FVM) using the QUICK differencing scheme, which is less dispersive and quite accurate. Simulations were performed using commercial CFD software FLUENT 15.1. The SIMPLE algorithm was used for the pressure-velocity coupling and a second-order implicit scheme was used for the transient formulation with a time step of $1 \times 10^{-3} \mathrm{~s}$. Since the turbulent length scales and grid dimensions varied in the present computational domain, the RANS computed value of the resolution control parameter $\left(f_{k}\right)$ employing Eq. (4) also varied in the computational domain. An optimum value of $f_{k}$ was therefore obtained by calculating the probability density function of $f_{k}$ in the computational domain to determine its value supported by the maximum grid points. The value of $f_{k}$ in PANS computations was kept larger than the RANS computed optimum value of $f_{k}$ to ensure that the modelled and resolved turbulent scales were in accordance with the grid dimensions. Alternatively, the $f_{k}$ value could be dynamically updated as described by Krajnović et al [19]. However, sufficient care should be taken to make sure that the $f_{k}$ value remains bounded between 0 and 1 . In the present study, the PANS computations were performed at two constant values of $f_{k}$ equal to 0.7 and 0.3 to assess the effect of variable resolution in the PANS methodology on the same computational grid. The value of $f_{\varepsilon}$ was taken to be 1 in both the PANS computations [15].

\section{Results and discussion}

For a fully developed turbulent Czochralski flow, timeaveraging over a long period on a sufficiently fine grid resolves the flow in well-defined characteristic recirculation cells arising due to the balance of mass, momentum and various body forces. These characteristic cells are described as [5] follows.

(i) Taylor-Proudman cell (A): Induced by the centrifugal force generated by the rotating crystal against the radial pressure gradient and enlarged by the buoyancy.

(ii) Bénard cell or buoyancy driven cell (B): Due to high temperature along the crucible walls, buoyant upward flow is developed close to the crucible walls and flowing inwards towards the rotating crystal.

(iii) Cell due to interaction of (i) and (ii) (C): Due to the low aspect ratio of the crucible in the present case, the buoyant Bénard cell $\mathbf{B}$ is not stable and breaks down into another cell $\mathbf{C}$ on interaction with the Taylor-Proudman cell A.

(iv) Cell due to Marangoni Convection (D): Vortex D formed near the melt free-surface close to the crucible boundary is due to changes in the surface tension on account of strong radial temperature gradient on the free-surface near the crucible walls.

(v) Ekman layer or viscous boundary layer for rotation (E): Thin circulating vortex E right below the crystal arises due to the centrifugal forces generated by the counter-rotating crystal and crucible.

Figure 2 shows the computed time-averaged (mean) and instantaneous stream-traces on a two-dimensional crosssectional plane using URANS and PANS (with $f_{k}=0.3$ and $\left.f_{k}=0.7\right)$ methods. It is observed that the URANS approach computes one major cell (A) covering the entire domain, which is actually formed by the combined TaylorProudman effect and buoyant forces. Hence the URANS computations do not clearly capture the buoyancy cell B and interacting cell $\mathrm{C}$. The URANS approach was also able to resolve cell $\mathrm{D}$, indicating strong Marangoni convection in the melt. Both the mean and the instantaneous streamtrace plots of the URANS computations are identical 

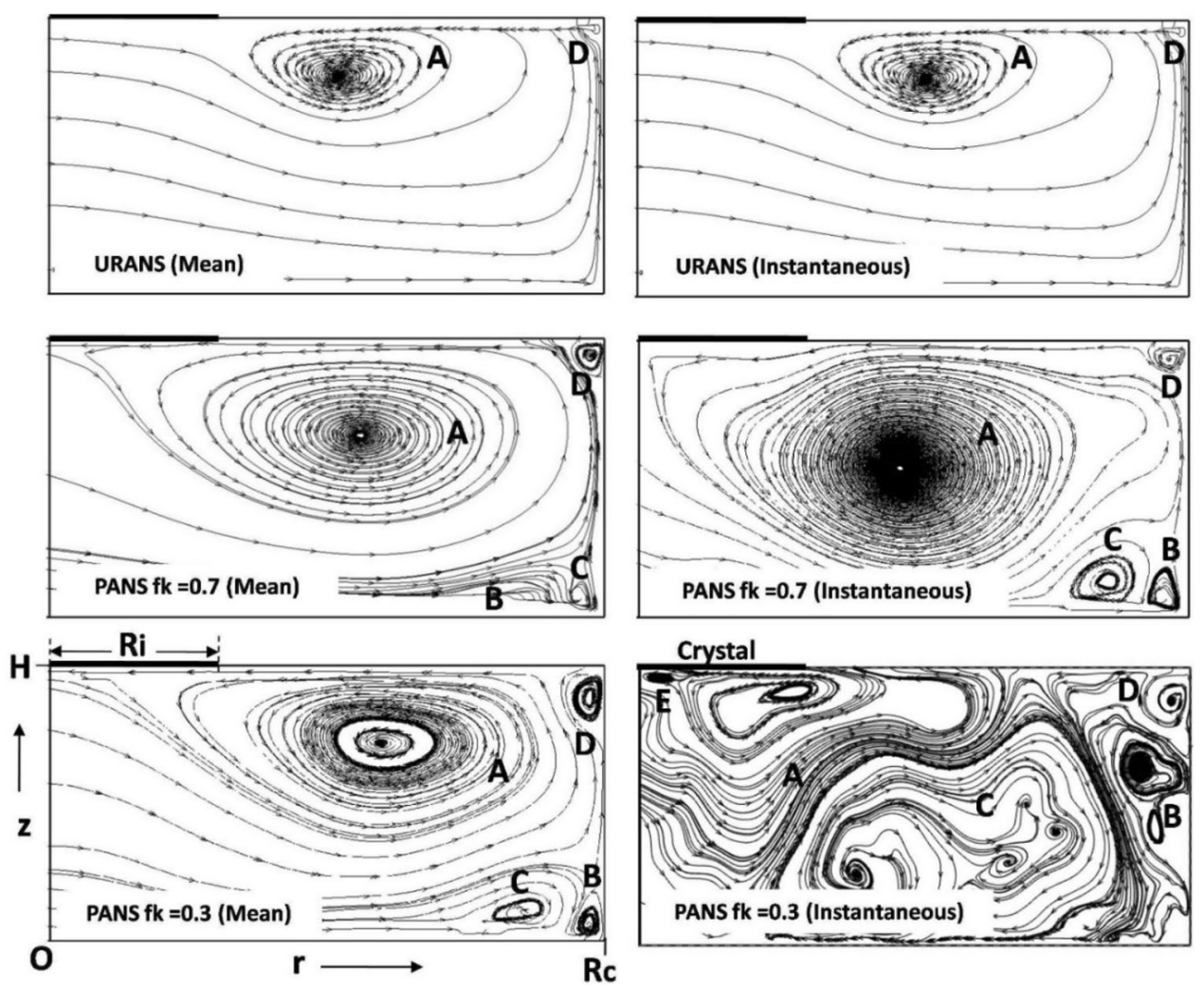

Figure 2. Comparison of stream-trace plots of URANS and PANS (with $f_{k}=0.7$ and $f_{k}=0.3$ ) computations on a vertical crosssectional plane (axis on left side and crucible wall on right side; $H, R_{c}$ and $R_{i}$ are the crucible height, crucible radius and crystal radius, respectively).

(figure 2), indicating that the URANS equations do not capture the unsteadiness of the flow in the present study. The PANS computations with $f_{k}=0.7$ resolve the flow into four cells A, B, C and D, as already discussed. These cells can be clearly identified in the instantaneous stream-trace contours (figure 2). Further, in the PANS approach with $f_{k}=0.7$ the buoyancy cell $\mathrm{B}$ and interacting cell $\mathrm{C}$ are restricted to a small volume at the bottom corner. However, according to the DNS results [5, 6] these cells should cover the complete height of the melt. Hence the PANS computations at $f_{k}=0.7$ do not accurately capture the cells in upper portion of the melt with relatively low buoyancy. The structure of the instantaneous stream-traces is also similar to that of the mean stream-traces, representing less fluctuations being captured with $f_{k}=0.7$. As the filter width is reduced to $f_{k}=0.3$ more turbulent scales are resolved, as confirmed by highly unsteady and disordered instantaneous stream-trace contours (figure 2). Cells A, B and C are of comparable size and show a vertical orientation in the instantaneous stream-traces plot (figure 2). This behaviour is consistent with the Czochralski melt flow behaviour. Cell $\mathrm{D}$ due to Marangoni convection can also be clearly identified in both the mean and instantaneous stream-trace contours with $f_{k}=0.3$. It can also be seen that a small cell $\mathrm{E}$ is deciphered in the instantaneous stream-trace plot with $f_{k}=0.3$, which is the Ekman layer cell. The same is not deciphered in the corresponding PANS computed mean flow (with $f_{k}=0.3$ ) and this behaviour may be due to the smearing effect of flow averaging. It is also to be noted that the flow in Czochralski melt is highly anisotropic due to concurrent effect of a number of forces and hence the instantaneous shapes and orientations of the characteristic cells continuously change. As highlighted by Raufeisen et al [5], time averaging for extremely long period of time followed by a circumferential averaging of the flow is required to remove the effect of flow anisotropy on the shape of these characteristic flow cells.

\section{Conclusion}

The PANS methodology has been applied for the analysis of an idealized Czochralski flow set-up. It is observed that in comparison with the URANS approach, the PANS method is able to capture the unsteadiness of fluctuations of the Czochralski flow with more characteristic recirculating cells being resolved with a decrease in the PANS filter width. The paper presents preliminary results on application of the PANS methodology to resolve the Czochralski flow structure. The PANS approach has shown good 
potential of resolving the flow characteristic of the Czochralski melt. To substantiate these results, a detailed study on physical and numerical aspects of suitability of the PANS methodology for modelling the Czochralski melt flow along with comparisons to the existing experimental, LES and DNS results is required. Such a study is being performed by the present authors.

\section{References}

[1] Ristorcelli J R and Lumley J L 1992 Instabilities, transition and turbulence in the Czochralski crystal melt. J. Cryst. Growth 116: 447-460

[2] Kakimoto K 1995 Flow Instability during crystal growth from the melt. Prog. Cryst. Growth Charact. 30: 191-215

[3] Enger S, Breuer M and Basu B 2000 Numerical simulation of fluid flow and heat transfer in an industrial Czochralski melt using a parallel-vector supercomputer. In: Krause E and Jäger W (Eds.) High Performance Computing in Science and Engineering '99. Berlin-Heidelberg: Springer-Verlag, pp. 253-266

[4] Raufeisen A, Breuer M, Botsch T and Delgado A 2009 LES validation of turbulent rotating buoyancy- and surface tension-driven flow against DNS. Comput. Fluids 38: 1549-1565

[5] Raufeisen A, Breuer M, Botsch T and Delgado A 2008 DNS of rotating buoyancy- and surface tension-driven flow. Int. J. Heat Mass Transfer 51: 6219-6234

[6] Wagner C and Friedrich R 2004 Direct numerical simulation of momentum and heat transport in idealized Czochralski crystal growth configurations. Int. J. Heat Fluid Flow 25: 431-443

[7] Kumar V, Biswas G, Brenner G and Durst F 2003 Effect of thermocapillary convection in an industrial Czochralski crucible: numerical simulation. Int. J. Heat Mass Transfer 46: $1641-1652$

[8] Verma S and Dewan A 2014 Solidification modeling: evolution, benchmarks, trends in handling turbulence, and future directions. Metall. Mater. Trans. B 45: 1456-1471

[9] Girimaji S S 2006 Partially-averaged Navier-Stokes model for turbulence: a Reynolds-averaged Navier-Stokes to direct numerical simulation bridging method. J. Appl. Mech. 73: 413-421

[10] Lakshmipathy S and Girimaji S S 2010 Partially averaged Navier-Stokes (PANS) method for turbulence simulations: flow past a circular cylinder. J. Fluids Eng. 132: 121202-1-121202-9

[11] Kumar R and Dewan A 2015 Partially-averaged NavierStokes method for turbulent thermal plume. Heat Mass Transfer 51: 1655-1667

[12] Razi P, Venugopal V, Jagannathan S and Girimaji S 2015 Partially averaged Navier-Stokes (PANS) simulations of liddriven cavity flow-part ii: flow structures. In: Girimaji S, Haase W, Peng S H and Schwamborn D (Eds.) Progress in hybrid RANS-LES modelling, Notes on numerical fluid mechanics and multidisciplinary design, vol. 30. Springer International Publishing, pp. 421-430

[13] Gräbner O, Müller G, Virbulis J, Tomzig E and Ammon W v 2001 Effects of various magnetic field configurations on temperature distributions in Czochralski silicon melts. $\mathrm{Mi}$ croelectron. Eng. 56: 83-88

[14] Enger S, Gräbner O, Müller G, Breuer M and Durst F 2001 Comparison of measurements and numerical simulations of melt convection in Czochralski crystal growth of silicon. $J$. Cryst. Growth 230: 135-142

[15] Girimaji S S and Abdol-Hamid K S 2005 Partially-averaged Navier-Stokes model for turbulence: implementation and validation. In: Proceedings of the 43rd AIAA Aerospace Sciences Meeting and Exhibit, Reno, NV, AIAA 2005, vol. 502, pp. $1-14$

[16] Maa J M, Peng S H, Davidson L and Wang F J 2011 A low Reynolds number variant of partially-averaged NavierStokes model for turbulence. Int. J. Heat Fluid Flow 32: 652-669

[17] Launder B E and Sharma B I 1974 Application of the energydissipation model of turbulence to the calculation of flow near a spinning disc. Lett. Heat Mass Transfer I: 131-138

[18] Lipchin A and Brown R A 1999 Comparison of three turbulence models for simulation of melt convection in Czochralski crystal growth of silicon. J. Cryst. Growth 205: 71-91

[19] Krajnović S, Minelli G and Basara B 2016 Partially-averaged Navier-Stokes simulations of two bluff body flows. Appl. Math. Comput. 272: 692-706 